\title{
Drug loaded magnetic nanoparticles for cancer therapy
}

\author{
R Jurgons ${ }^{1}$, C Seliger ${ }^{1}$, A Hilpert ${ }^{2}$, L Trahms ${ }^{3}$, S Odenbach ${ }^{4}$ and \\ C Alexiou ${ }^{1}$ \\ ${ }^{1}$ Department of Otorhinolaryngology, Head and Neck Surgery, University Erlangen-Nürnberg, \\ Germany \\ 2 Department of Anatomy I, University Erlangen-Nürnberg, Germany \\ ${ }^{3}$ Physikalisch-Technische Bundesanstalt, Berlin, Germany \\ ${ }^{4}$ Lehrstuhl für Magnetofluiddynamik, Universität Dresden, Germany \\ E-mail: roland_jurgons@web.de and C.Alexiou@web.de
}

Received 29 April 2006, in final form 14 July 2006

Published 8 September 2006

Online at stacks.iop.org/JPhysCM/18/S2893

\begin{abstract}
Magnetic nanoparticles have been investigated for biomedical applications for more than 30 years. In medicine they are used for several approaches such as magnetic cell separation or magnetic resonance imaging (MRI). The development of biocompatible nanosized drug delivery systems for specific targeting of therapeutics is the focus of medical research, especially for the treatment of cancer and diseases of the vascular system. In an experimental cancer model, we performed targeted drug delivery and used magnetic iron oxide nanoparticles, bound to a chemotherapeutic agent, which were attracted to an experimental tumour in rabbits by an external magnetic field (magnetic drug targeting). Complete tumour remission could be achieved. An important advantage of these carriers is the possibility for detecting these nanoparticles after treatment with common imaging techniques (i.e. x-ray-tomography, magnetorelaxometry, magnetic resonance imaging), which can be correlated to histology.
\end{abstract}

\section{Introduction}

Magnetic nanoparticles may open up a wide field of possible applications in medicine. Up to now, magnetic nanoparticles have been used in medicine for magnetic separation techniques [1-9] as contrast agents in magnetic resonance imaging [10-13], for local hyperthermia [14-17] or as magnetic targetable carriers for several drug delivery systems [18-21].

For application in biological organisms, these particles have to be biocompatible. It could be shown that, generally, starch-coated magnetic iron oxide nanoparticles are tolerated quite well $[22,23]$. Because of this, and because of the fact that these kinds of particles are produced as commercial contrast agents for MRI investigations (Combidex ${ }^{\circledR}$, Resovist $^{\circledR}$, 


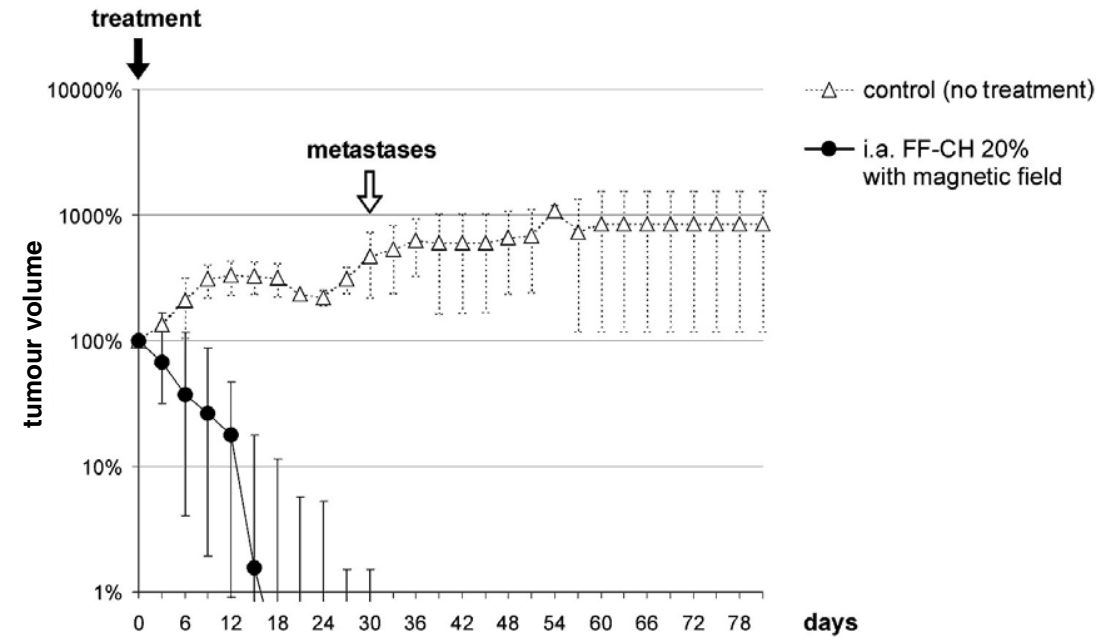

Figure 1. Chemotherapeutic treatment with magnetic drug targeting on tumour-bearing rabbits. Complete tumour remission could be achieved with only $20 \%$ of the regular systemic dose: therapy group, $n=5$; control group, $n=2$ [28].

Endorem ${ }^{\circledR}$, Sinerem ${ }^{\circledR}$ ), iron oxide nanoparticles seem to be a convenient carrier material for drug targeting systems. This class of particles show superparamagnetic properties and can be attracted by an external magnetic field. Furthermore, nanosized particles are small enough to prevent occlusions of the vascular system, especially the capillaries. The required size of the particles probably has to be smaller than $5 \mu \mathrm{m}$ [24]. On the other hand, magnetic nanoparticles should not be too small, because of their attractability by an external magnetic field gradient. Investigations of the size-dependent body distribution of these particles have shown that particles larger than $100 \mathrm{~nm}$ in diameter are intercepted more by cells of the MPS (mononuclear phagocyte system) than smaller particles [25]. After intravenous application (the regular administration of parenteralia), the magnetic particles can be found in macrophages of the liver, spleen and lung, because of the so-called first-pass effect [26, 27].

To avoid this effect, we performed our approach for local cancer chemotherapy with starchcoated iron oxide nanoparticles, dispersed in deionized water, with intra-arterial application into the tumour-supplying artery. The magnetic particles were coupled to the chemotherapeutic agent mitoxantrone and attracted and held in the tumour region by a strong external magnetic field gradient after application (magnetic drug targeting). In previous studies, it could be shown that this drug delivery system can cause complete tumuor remission in tumour-bearing rabbits [28-31]. In addition to that, the applied dose of the drug could be diminished to $20 \%$ of the regular systemic dose. Tumour remission was achieved without any negative side-effects, which are common after regular cancer chemotherapy (figure 1). These results indicate the great advantage of this magnetically guided drug delivery system, which is caused by the reduction of the given chemotherapeutic agent and the focused application protecting healthy tissue [21, 32-35].

Investigations of the distribution of the particles showed a high accumulation of magnetic particles in the tumuor region, in contrast to other body compartments (i.e. liver or spleen). This was performed by measurements of ${ }^{59} \mathrm{Fe}$-nanoparticles, $\mathrm{x}$-ray tomography pictures and magnetorelaxometry of the relaxation signal by superconducting quantum interference detector (SQUID) sensors in correspondence to histological examinations [27, 36, 37]. 


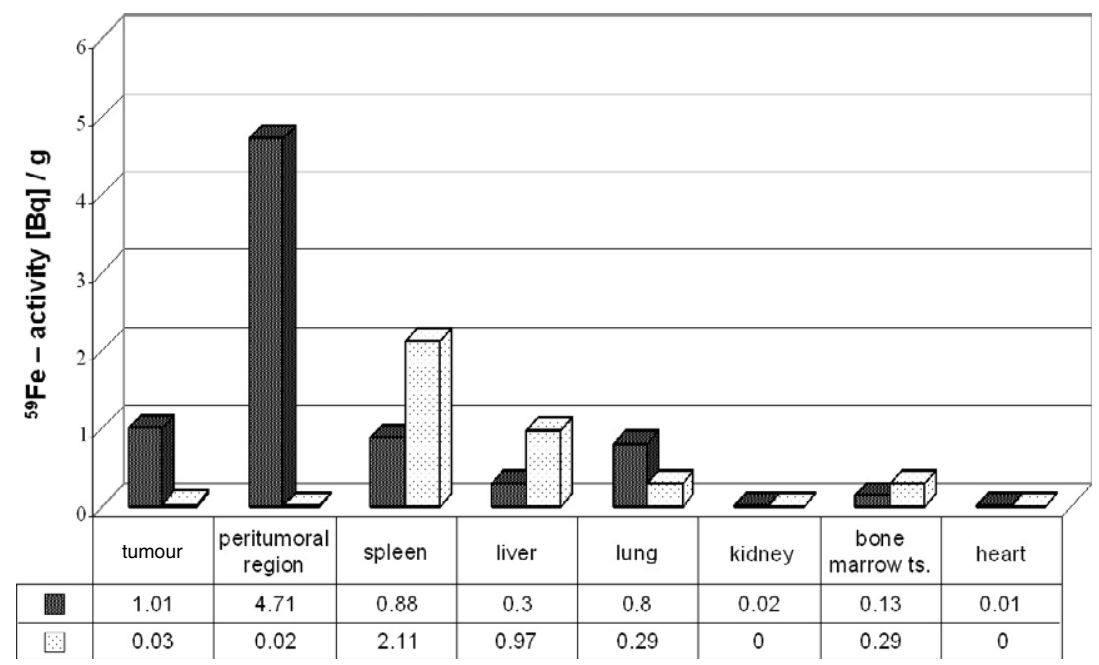

Figure 2. Radioactive ${ }^{59} \mathrm{Fe}$-distribution $60 \mathrm{~min}$ after intra-arterial ${ }^{59} \mathrm{Fe}$-ferrofluid application; 眲 i.a. ${ }^{59} \mathrm{Fe}$-ferrofluids with external magnetic field $(n=2)$; $\square$ i.a. ${ }^{59} \mathrm{Fe}$-ferrofluids without external magnetic field $(n=1)$; ts. = tumor side [27].

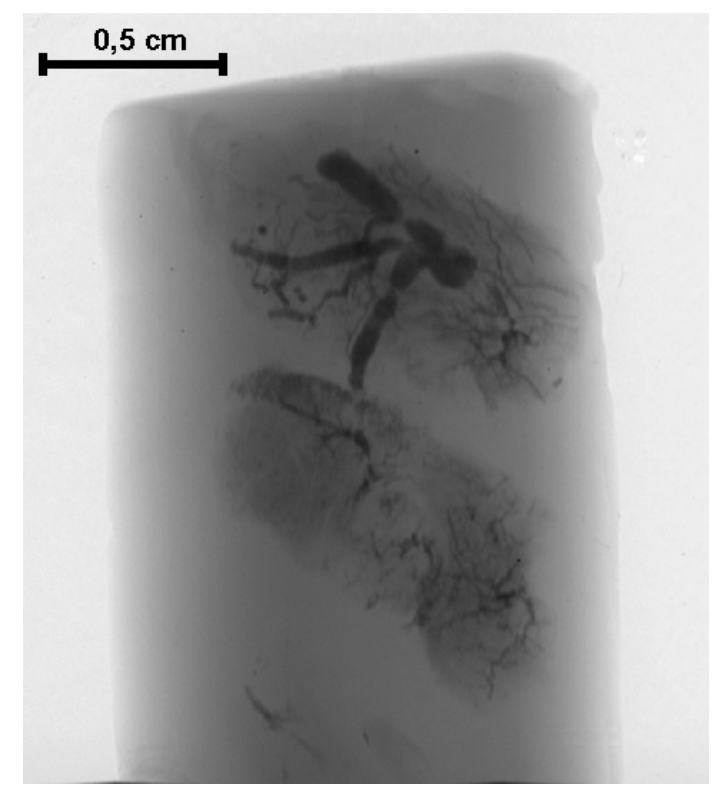

Figure 3. X-ray picture of a VX2-tumour tissue sample embedded in paraffin after magnetic drug targeting (MDT). The nanoparticles are visible in the vascular system of the tumour [36].

Investigations concerning the particle distribution showed a high accumulation of the magnetic particles in the tumour region in contrast to other body compartments, i.e. liver or spleen. In experiments using radioactive ${ }^{59} \mathrm{Fe}$-nanoparticles, 114 times more activity could be detected in the tumour region after magnetic drug targeting compared to the control without a magnetic field [27] (figure 2).

$\mathrm{X}$-ray-tomography pictures show the iron oxide nanoparticles after magnetic drug targeting in the vascular system of the tumour (figure 3).

The corresponding histological cross sections show the nanoparticles after intra-arterial application in the vascular system of the tumour (figure 4). SQUID-based magnetorelaxometry 


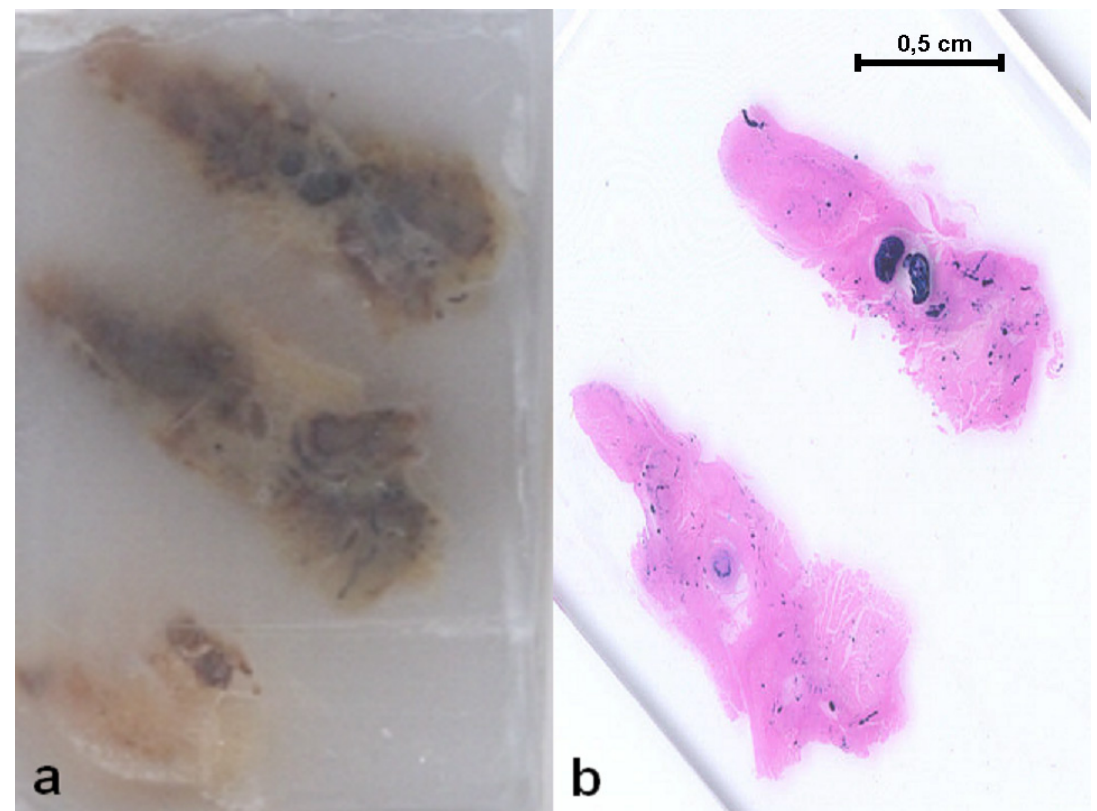

Figure 4. (a) VX2-tumour tissue after magnetic drug targeting embedded in paraffin. (b) Synoptic picture of the corresponding histological slide stained with Prussian blue. The nanoparticles are visible as blue pigment in the vessels of the tumour [36].
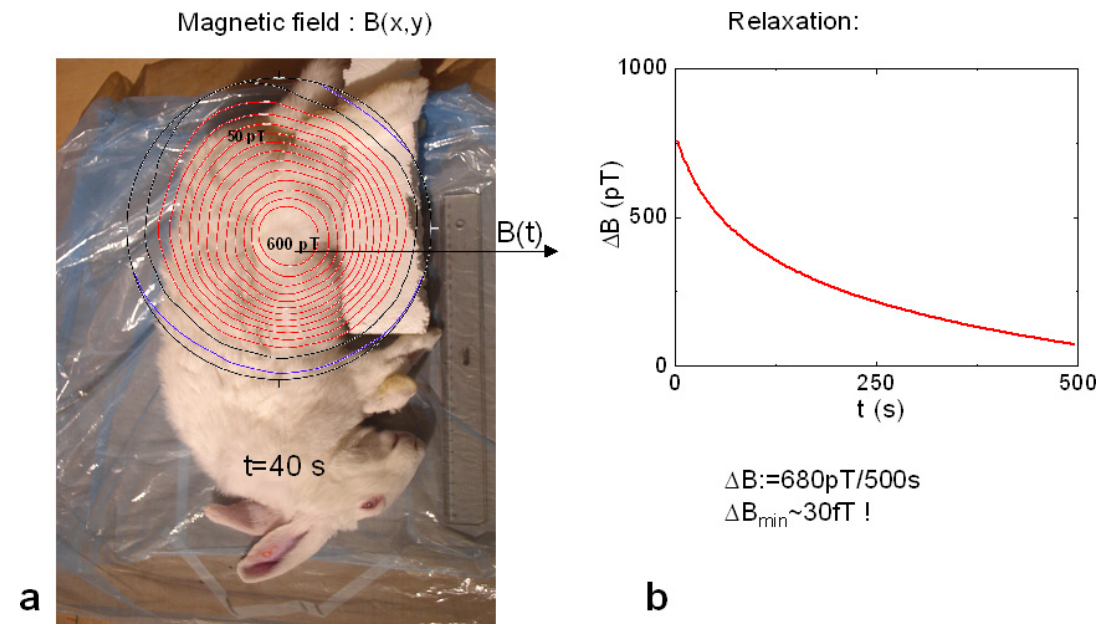

$\Delta \mathrm{B}:=680 \mathrm{pT} / 500 \mathrm{~s}$

$\Delta \mathrm{B}_{\min } \sim 30 \mathrm{fT}$ !

b

Figure 5. (a) Magnetic field distribution of magnetic nanoparticle relaxation after magnetization. The centre of the circular-shaped field distribution corresponds to the tumour position. (b) Single channel relaxation signal of the magnetic nanoparticles [37].

measurements of the relaxation signal of the nanoparticles proved non-invasively that a high amount of magnetic nanoparticles $(\sim 85 \%)$ was accumulated in the tumour region, together with a much smaller accumulation in the liver region [37] (figure 5). The observed slow time decay of the magnetization (figure 5(b)) was attributed to Néel relaxation, indicating that the particles in the tumour were immobilized. 

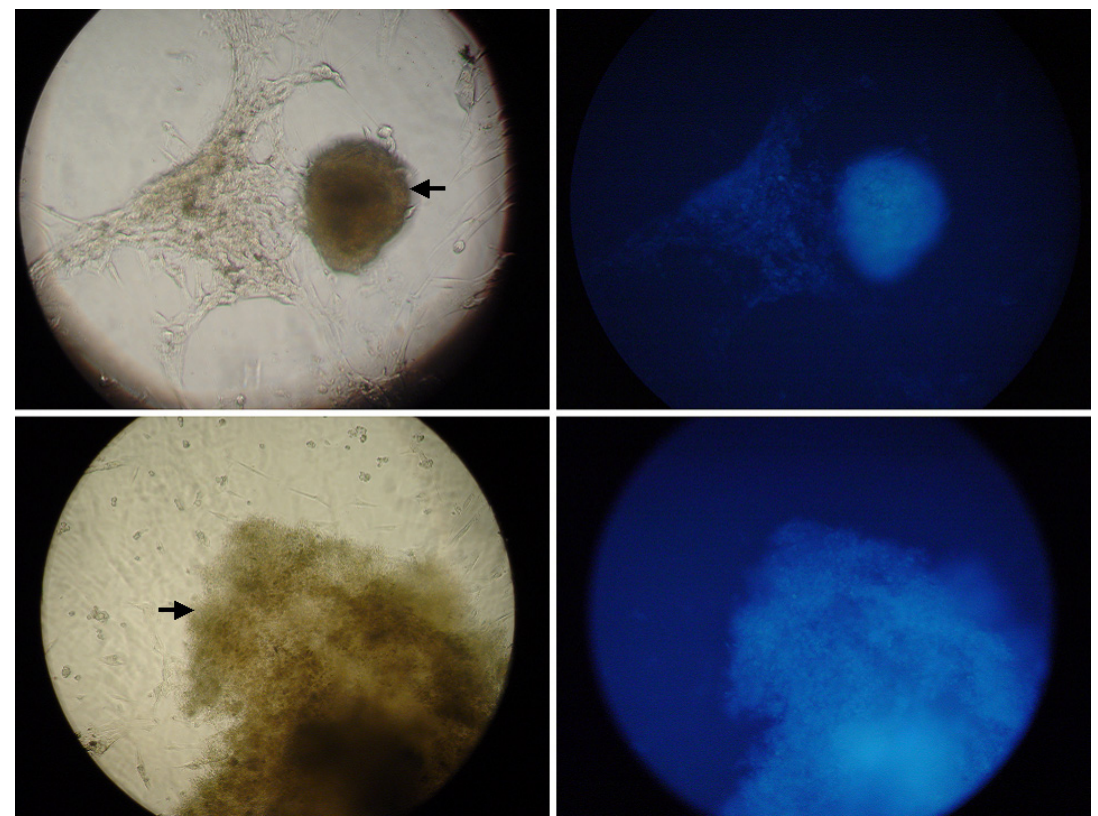

Figure 6. Proof of DNA-intercalated mitoxantrone in cultivated VX2 squamous cell carcinoma cells by fluorescence microscopy. Left side: phase contrast microscopy pictures of the cell culture. Right side: fluorescence pictures of the same areas; arrows: tumor cell conglomerates; magnification: $200 \times[38]$.

Additionally the distribution of the chemotherapeutic drug which is the therapeutic agent in this cancer treatment was investigated [27].

In vitro studies with fluorescence labelling of tumour cell culture have shown the direct binding of mitoxantrone to the cell nucleus, the required place of drug interaction [38].

Comparing different application modes, high pressure liquid chromatography analysis of the chemotherapeutic agent after magnetic drug targeting proved a 75 times higher concentration of the chemotherapeutic agent in the tumour region compared to the regular systemic administration $[27,39,40]$.

In VX2 tumor cell culture incubated with mitoxantrone for one hour, only DNA-coupled mitoxantrone in the tumour cells was fluorescence positive (figure 6). Fibroblast-like cells in the cell culture do not show any fluorescence [38].

Despite in vitro and animal experiments, it is necessary that nanoparticles for in vivo applications in human patients need to be manufactured and analysed under the guidelines of pharmaceutical regulations. This is realized for commercially available iron oxide nanoparticle suspensions used as contrast agent in MRI. But these nanoparticle suspensions have to be administered strictly intravenously and highly diluted in deionized water to avoid embolization caused by the loss of the colloidal stability and the formation of bigger conglomerates [24]. The coating and the charge state of nanoparticle suspensions are responsible for their compatibility in the biological organism [26, 41]. With the application of an external magnetic field after intra-arterial drug delivery, a local accumulation of nanoparticles occurs with a potential increase in conglomerate formation and the risk of vessel occlusions.

Therefore we investigated the colloidal stability of the iron oxide nanoparticles used in our experiments under conditions of different physiological and injectable solutions by particle sizing, measurements of zeta potential, electron microscopy and with an in vitro artery model. 


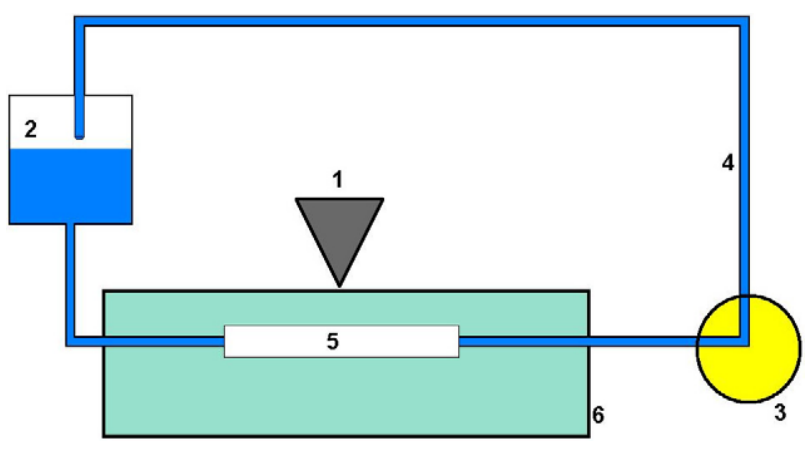

Figure 7. Schematic drawing of the artery model: 1 , top of the pole shoe; 2 , reservoir of the circulating fluid; 3 , pump; 4, circulation; 5 , artery; 6 , buffer.

\section{Material and methods}

\subsection{Magnetic nanoparticles}

The nanoparticles were manufactured by Chemicell (Berlin, Germany) and consist of iron oxides covered by phosphated starch polymers for colloidal stabilization in deionized water.

\subsection{Magnetic field}

For the experiments, we used a powerful electromagnet with a maximum magnetic field strength of $1.7 \mathrm{~T}$ and a magnetic field gradient of $10 \mathrm{~T} \mathrm{~m}^{-1}$.

\subsection{Mitoxantrone (MTX)}

The chemotherapeutic agent that was used, coupled to the magnetic nanoparticles, is the anthracendion derivative MTX-HCL (Mitoxantrone, Novantron ${ }^{\circledR}$; Lederle, Wyeth-Pharma, Germany). It inhibits DNA and RNA syntheses and causes DNA-strand breaks by intercalation.

\subsection{Colloidal stability}

2.4.1. Particle sizing. Particle suspension were diluted 1:100 with distilled water. The size was determined with dynamic laser scattering (DLS, Nicomp 380 ZLS, Santa Barbara, CA, USA). Further DLS investigations were performed with the same dilution in $0.9 \%$ $\mathrm{NaCl}$ solution, Ringer buffer and physiological electrolyte solution (Tutofosin) to simulate physiological or probable injection parameters.

2.4.2. Zeta potential. Zeta-potential measurements were performed with the Zeta option of the DLS equipment. Dilutions were the same compared to DLS measurements. Additional to the above-mentioned conditions, the zeta potential was determined in electrolyte-albumine solution (bovine serum albumine, Sigma, Germany) as a potential serum substitute.

2.4.3. Electron microscopy. Ferrofluids in different solutions (distilled water, $0.9 \% \mathrm{NaCl}$ ) were fixed in 2\% Agar (Merck, Darmstadt, Germany). After dehydration with ethanol (Merck) and acetone, the samples were embedded in Epon (Epoxyd resin, Roth, Karlsruhe, Germany).

2.4.4. Artery model. For this model, we used freshly isolated bovine femoral arteries. The artery is mounted in a tempered circuit (figure 7). As a flow medium, we used Krebs-Ringer 
Table 1. Volume-weighted size determination by DLS under different dilution conditions.

\begin{tabular}{lll}
\hline $\begin{array}{l}\text { Different batches of } \\
\text { nanoparticle suspensions }\end{array}$ & $\begin{array}{l}\text { Medium particle size in } \\
\mathrm{H}_{2} \mathrm{O}\end{array}$ & $\begin{array}{l}\text { Medium particle size in } \\
0.9 \% \mathrm{NaCl}\end{array}$ \\
\hline 1 & $125.0 \mathrm{~nm}(45.5 \%)$ & $207.6 \mathrm{~nm}(4.2 \%)$ \\
& $345.4 \mathrm{~nm}(54.5 \%)$ & $1897.4 \mathrm{~nm}(95.8 \%)$ \\
2 & $80.0 \mathrm{~nm}(80.4 \%)$ & $137.2 \mathrm{~nm}(5.3 \%)$ \\
& $207.3 \mathrm{~nm}(19.6 \%)$ & $2023.3 \mathrm{~nm}(94.7 \%)$ \\
3 & $81.5 \mathrm{~nm}(65.9 \%)$ & $107.1 \mathrm{~nm}(7.8 \%)$ \\
& $280.3 \mathrm{~nm}(34.1 \%)$ & $2958.7 \mathrm{~nm}(92.2 \%)$ \\
4 & $76.3 \mathrm{~nm}(86.4 \%)$ & $77.8 \mathrm{~nm}(7.4 \%)$ \\
5 & $188.8 \mathrm{~nm}(13.6 \%)$ & $6191.3 \mathrm{~nm}(92.6 \%)$ \\
& $178.4 \mathrm{~nm}(57.1 \%)$ & $110.9 \mathrm{~nm}(14.2 \%)$ \\
& $471.9 \mathrm{~nm}(42.9 \%)$ & $1279.2 \mathrm{~nm}(85.8 \%)$ \\
\hline
\end{tabular}

Table 2. Zeta-potential determinations of different nanoparticle suspensions corresponding to table 1.

\begin{tabular}{lcrrr}
\hline $\begin{array}{l}\text { Different produced } \\
\text { nanoparticle suspensions }\end{array}$ & $\mathrm{H}_{2} \mathrm{O}$ & $0.9 \% \mathrm{NaCl}$ & Tutofosin & Tutofosin/BSA \\
\hline 1 & $-28.79 \mathrm{mV}$ & $-1.77 \mathrm{mV}$ & $0.58 \mathrm{mV}$ & $1.22 \mathrm{mV}$ \\
2 & $-34.47 \mathrm{mV}$ & $-2.65 \mathrm{mV}$ & $0.43 \mathrm{mV}$ & Not measured \\
3 & $-28.07 \mathrm{mV}$ & $0.32 \mathrm{mV}$ & $-0.22 \mathrm{mV}$ & $-0.34 \mathrm{mV}$ \\
4 & $-38.29 \mathrm{mV}$ & $-0.73 \mathrm{mV}$ & $-0.83 \mathrm{mV}$ & $0.23 \mathrm{mV}$ \\
5 & $-28.59 \mathrm{mV}$ & $-3.16 \mathrm{mV}$ & $1.01 \mathrm{mV}$ & $0.90 \mathrm{mV}$ \\
\hline
\end{tabular}

buffer $\mathrm{pH} 7.4$ substituted with $0.625 \%$ albumine. The artery was placed near the tip of the electromagnet. Nanoparticle suspensions were applied by a side inlet under pulsatile flow.

Using a pump, it is possible to simulate different flow velocities and different vessel pressures.

After the magnetization procedure, the outflow was collected. Differences in particle agglomerate diameter were measured by DLS.

\section{Results}

We detected an increasing instability of the particle suspension in the presence of physiological salt solutions in the size determination by DLS (table 1) and with zeta-potential measurements (table 2) in comparison to measurements in deionized water.

The results could be confirmed by electron microscopy pictures of the particles suspended in deionized water versus physiological $0.9 \% \mathrm{NaCl}$ (figure 8 ).

The same phenomenon was observed in the artery model. The decrease in the zeta potential in the physiological surrounding (Krebs-Ringer buffer pH 7.4 substituted with $0.625 \%$ albumine), combined with the applied magnetic field, leads to total decomposition of the nanoparticle suspension (figure 9).

\section{Conclusions and discussion}

Investigations of the distribution of the magnetic nanoparticles after intra-arterial infusion and magnetic targeting showed that this drug delivery system is suitable for attracting magnetic iron oxide particles to a respective body compartment (i.e. tumour). This was examined after 


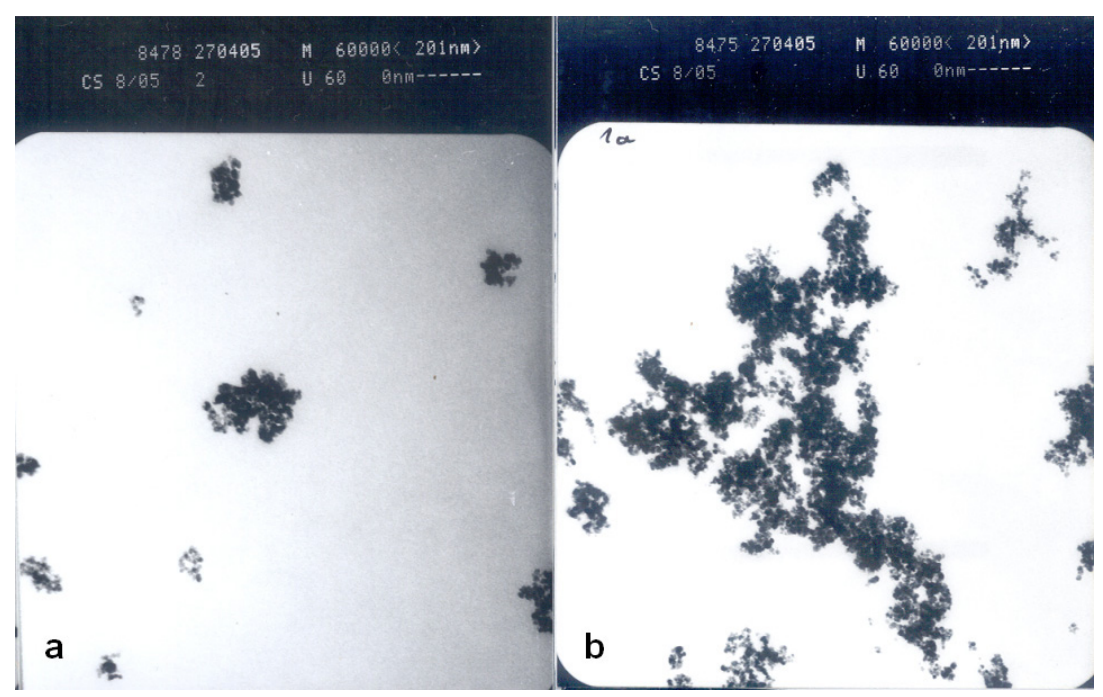

Figure 8. Electron microscopy of the same nanoparticle suspension in deionized water (a) and in $0.9 \% \mathrm{NaCl}$ (b).

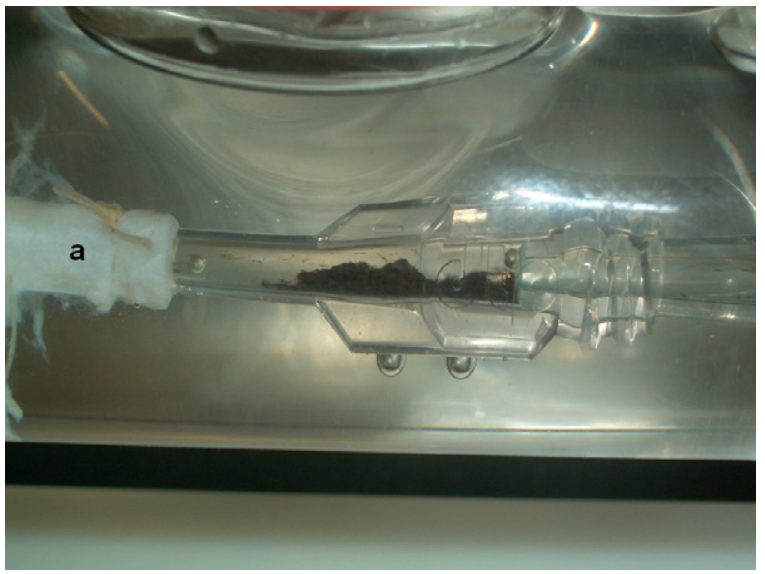

Figure 9. Artery model: a precipitate of nanoparticles in the circuit after the application of magnetic force at the outlet of the artery (a).

an extensive manufacturing procedure with radioactive ${ }^{59} \mathrm{Fe}$-particles. In a pilot study, we could show that this is also possible non-invasively with common imaging techniques like x-ray tomography and magnetorelaxometry. The results of these imaging techniques were confirmed by histological cross sections. This allows the control of a successful particle accumulation directly after treatment with this form of local chemotherapy.

HPLC studies of tissue samples proved that the distribution of the chemotherapeutic agent follows distribution of the applied nanoparticles and excludes the assumption that the chemotherapeutic agent desorbs too early before the drug carrier reaches the site of action (i.e tumour region). In addition to that, we demonstrated the direct binding of the chemotherapeutic agent in tumour cells with fluorescence detection of the intercalated DNA.

The efficacy of this treatment was only observed after intra-arterial application of the nanoparticles and not after intravenous particle administration [27]. 
Due to the intra-arterial application of magnetic nanoparticle suspensions, other facts have to be taken into consideration. All intra-arterial interventions are associated with a potential thrombotic risk, more than after intravenous application. In previous studies, we already had indications for a partial defragmentation of ferrofluid suspensions in vivo due to particle enrichment in the lung [27]. Two possible mechanisms for a thrombotic event after intra-arterial delivery are imaginable. First, the surface of the particle suspension causes a direct activation of the thrombotic system. This mechanism is also present after intravenous application and depends on the interaction of the particle surface with the activators of the coagulation cascade.

Second, instability of particle solution with the formation of greater agglomerates may lead to an occlusion of capillaries proximal to the application side and a subsequent occlusion of the artery, which may lead to life-threatening situations. This mechanism is much more relevant after intra-arterial application. Therefore nanoparticle suspensions for tumour treatment in humans have to be stable under physiological conditions.

To achieve pharmaceutically acceptable drug delivery systems for this promising therapeutic concept, further modifications of the particle surface and colloidal stability are necessary to apply this cancer therapy in human patients.

\section{Acknowledgments}

These studies were supported by the DFG (Deutsche Forschungsgemeinschaft) SPP1104 and in part by the BMBF (Bundesministerium für Bildung und Forschung).

\section{References}

[1] Stechell C H 1985 Magnetic separations in biotechnology—a review J. Chem. Technol. Biotechnol. 35B 175

[2] Olsvik O, Popovic T, Skjerve E, Cudjoe K S, Hornes E, Ugelstad J and Uhlen M 1994 Magnetic separation techniques in diagnostic microbiology Clin. Microbiol. Rev. 7 43-54

[3] Safarikova M and Safarik I 2001 Immunomagnetic separation of Escherichia coli O26, O111 and O157 from vegetables Lett. Appl. Microbiol. 33 36-9

[4] Deponte S, Steingroewer J, Löser C, Boschke E and Bley T 2004 Biomagnetic separation of Escherichia coli by use of anion-exchange beads: measurement and modeling of the kinetics of cell-bead interactions Anal. Bioanal. Chem. 379 419-26

[5] Durcova-Hills G, Tokunaga T, Kurosaka S, Yamaguchi M, Takahashi and Imai H 1999-2000 Immunomagnetic isolation of primordial germ cells and the establishment of embryonic germ cell lines in the mouse Cloning $1217-24$

[6] Brooimans R A, de Leeuw N, Bontenbal M and Gratama J W 2005 An immunomagnetic epithelial tumor cell enrichment model for minimal residual disease detection of cytokeratin $8+$ malignancies J. Biol. Regul. Homeost. Agents. 19 84-91

[7] Varshney M, Yang L, Su X L and Li Y 2005 Magnetic nanoparticle-antibody conjugates for the separation of Escherichia coli $\mathrm{O} 157: \mathrm{H} 7$ in ground beef J. Food. Prot. 68 1804-11

[8] Lekowska-Kochaniak A, Czajkowska D and Kokowski J 2002 Detection of Escherichia coli O157:H7 in raw meat by immunomagnetic separation and multiplex PCR Acta Microbiol. Pol. 51 327-37

[9] Safarik I and Safarikova M 1999 Use of magnetic techniques for the isolation of cells J. Chromatogr. B 722 33-5

[10] Stark D D, Weissleder R, Elizondo G, Hahn P F, Saini S, Todd L E, Wittenberg J and Ferucci J T 1988 Superparamagnetic iron oxide: clinical application as a contrast agent for MR imaging of the liver Radiology $168297-301$

[11] Suzuki M, Honda H, Kobayashi T, Wakabayashi T, Yoshida J and Takahashi M 1996 Development of a targetdirected magnetic resonance contrast agent using monoclonal antibody-conjugated magnetic particles Brain Tumor Pathol. 13 127-32

[12] Weissleder R, Cheng H C, Bogdanova A and Bogdanov A 1997 Magnetically labeled cells can be detected by MR imaging J. Magn. Reson. Imaging 7 258-63

[13] Pouliquen D, Perdrisot R, Ermias A, Akoda S, Jallet P and Jeune J J 1989 Superparamagnetic iron oxide nanoparticles as a liver MRI contrast agent: contribution of microencapsulation to improved biodistribution Magn. Reson. Imaging 7 619-27 
[14] Hilger I, Hiergeist R, Hergt R, Winnefeld K, Schubert H and Kaiser W A 2002 Thermal ablation of tumors using magnetic nanoparticles: an in vivo feasibility study Invest. Radiol. 37 580-6

[15] Jordan A, Wust P, Scholz R, Tesch B, Fähling H, Mitrovics T, Vogl T, Cervos-Navarro J and Felix R 1996 Cellular uptake of magnetic fluid particles and their effects on human adenocarcinoma cells exposed to AC magnetic fields in vitro Int. J. Hyperthermia 12 705-22

[16] Jordan A, Scholz R, Wust P, Fähling H and Felix R 1999 Magnetic fluid hyperthermia (MFH): Cancer treatment with AC magnetic field induced excitation of biocompatible superparamagnetic nanoparticles J. Magn. Magn. Mater. 201 413-9

[17] Jordan A, Scholz R, Maier-Hauff K, van Landeghem F K, Waldoefner N, Teichgraeber U, Pinkernelle J, Bruhn H, Neumann F, Thiesen B, von Deimling A and Felix R 2005 The effect of thermotherapy using magnetic nanoparticles on rat malignant glioma J. Neurooncol. $291-8$

[18] Kubo T, Sugita T, Shimose S, Nitta Y, Ikuta Y and Murakami T 2000 Targeted delivery of anticancer drugs with intravenously administered magnetic liposomes in osteosarcoma-bearing hamsters Int. J. Oncol. 17 309-15

[19] Alksne J F, Fingerhut A and Rand R 1966 Magnetically controlled metallic thrombosis of intracranial aneurysms Surgery $60212-8$

[20] Ito A, Shinkai M, Honda H and Kobayashi T 2005 Medical application of functionalized magnetic nanoparticles J. Biosci. Bioeng. 100 1-11

[21] Torchilin V P 2000 Drug targeting Eur. J. Pharm. Sci. 11 81-91

[22] Gao Y 2005 Biofunctionalization of magnetic nanoparticles Biofunctionalization of Nanomaterials ed C S Kumar (Winheim: Wiley-VCH) pp 72-98

[23] Tiefenauer L X, Tschiky A, Kühne G and Andres R Y 1996 In vivo evaluation of magnetite nanoparticles for use as a tumor contrast agent in MRI Magn. Reson. Imaging 14 391-402

[24] Müller R H, Jakobs C and Kayser O 2001 Nanosuspensions as particulate drug formulations in therapy-Rational for development and what we can expect for the future Adv. Drug Deliv. Rev. 47 3-19

[25] Sachdeva M S 1998 Drug targeting systems for cancer therapy Expert Opin. Invest. Drugs. 7 1849-64

[26] Storm G, Belliot S O, Daemen T and Lasic D D 1995 Suface modification of nanoparticles to oppose uptake by the mononuclear phagocyte system Adv. Drug Deliv. Rev. 17 31-48

[27] Alexiou C, Jurgons R, Schmid R, Bergemann C, Henke J, Erhardt W, Huenges E and Parak F G 2003 Magnetic drug targeting-biodistribution of the magnetic carrier and the chemotherapeutic agent Mitoxantron after locoregional cancer treatment J. Drug Target. 11 139-49

[28] Alexiou C, Arnold W, Klein R, Parak F G, Hulin P, Bergemann C, Erhardt W, Wagenpfeil S and Lübbe A S 2000 Locoregional cancer treatment with magnetic drug targeting Cancer Res. 60 6641-8

[29] Alexiou C, Arnold W, Hulin P, Klein R J, Renz H, Parak F G, Bergemann C and Lübbe A S 2001 Magnetic mitoxantrone nanoparticle detection by histology, x-ray and MRI after magnetic drug targeting J. Magn. Magn. Mater. 225 187-93

[30] Alexiou C, Arnold W, Hulin P, Klein R, Schmidt A, Bergemann C and Parak F G 2001 Therapeutic efficacy of ferrofluid bound anticancer agent Magnetohydrodynamics 37 318-22

[31] Alexiou C, Schmid R J, Jurgons R, Kremer M, Wanner G, Bergemann C, Huenges E, Nawroth T, Arnold W and Parak F G 2006 Targeting cancer cells: magnetic nanoparticles as drug carriers Eur. Biophys. J. 35 446-50

[32] Collins J M 1984 Pharmacological rationale for regional drug delivery J. Clin. Oncol. 2 498-505

[33] Gupta P K and Hung C K 1990 Comparative disposition of adriamycin delivered via magnetic albumin microspheres in presence and absence of magnetic fields in rats Life Sci. 46 471-84

[34] Gupta P K and Hung C K 1990 Targeted delivery of low dose doxorubicin hydrochloride administered via magnetic albumin mirospheres in rats J. Microencapsul. 7 85-92

[35] Gupta P K 1990 Drug targeting in chemotherapy: a clinical perspective J. Pharm. Sci. 79 949-62

[36] Alexiou C, Jurgons R, Seliger C, Brunke O, Iro H and Odenbach S 2006 Anticancer Res. at press

[37] Wiekhorst F, Jurgons R, Eberbeck D, Sander T, Steinhoff U, Hartwig S, Alexiou C and Trahms L 2005 SQUID system with integrated superconducting shield for monitoring of drug targeting with magnetic nanoparticles in animals Biomed. Tech. 50 609-10

[38] Alexiou C, Jurgons R, Seliger C, Kolb S, Heubeck B and Iro H 2006 Fluorescence microscopic investigations on VX2 squamous cell carcinoma cells Z. Phys. Chem. $220235-40$

[39] Alexiou C, Jurgons R, Schmid R, Hilpert H, Bergemann C, Parak F G and Iro H 2005 In vitro and in vivo investigations of targeted chemotherapy with magnetic particles J. Magn. Magn. Mater. 293 389-93

[40] Alexiou C, Jurgons R, Schmid R, Erhardt W, Parak F G, Bergemann C and Iro H 2005 Magnetisches drug targeting-ein neuer ansatz in der lokoregionären tumortherapie mit chemotherapeutika HNO 53 618-22

[41] Brigger I, Morizet J, Laudani L, Aubert G, Appel M, Velasco V, Terrier-Lacombe M J, Desmaele D, d'Angelo J, Couvreur P and Vassal G 2004 Negative preclinical results with stealth nanospheres-encapsulated Doxorubicin in an orthotopic murine brain tumor model J. Control. Release $10029-40$ 